\title{
Magnetocentrifugal jets and chondrule formation in protostellar disks
}

\author{
Raquel Salmeron $^{1,2}$ and Trevor Ireland ${ }^{2}$ \\ ${ }^{1}$ Research School of Astronomy \& Astrophysics, The Australian National University, \\ Weston Creek, ACT 2611, Australia; email: raquel@mso.anu.edu.au \\ ${ }^{2}$ Research School of Earth Sciences, The Australian National University, \\ Canberra, ACT 0200, Australia; email: trevor.ireland@anu.edu.au
}

\begin{abstract}
Chondrite meteorites are the building blocks of the solar nebula, out of which our Solar System formed. They are a mixture of silicate and oxide objects (chondrules and refractory inclusions) that experienced very high temperatures, set in a matrix that remained cold. Their prevalence suggests that they formed through a very general process, closely related to stellar and planet formation. However the nature and properties of the responsible mechanism have remained unclear. The evidence for a hot solar nebula provided by this material seems at odds with astrophysical observations of forming stars. These indicate that the typical temperatures of protostellar disks are too low to melt and vapourise silicate minerals at the radial distances sampled by chondrule-bearing meteorites. Here, we show that processing of precursors in a protostellar outflow at radial distances of about $1-3 \mathrm{AU}$ can heat them to their melting points and explain their basic properties, while retaining association with the colder matrix.
\end{abstract}

Keywords. solar system: formation, planetary systems: protoplanetary disks, ISM: jets and outflows

Protostellar winds are common in star-forming regions (e.g. Bally et al. 2007). The ubiquity of these winds and the correlation between accretion and outflow signatures in young stars (e.g. Cabrit 2007) suggest that they are an integral part of the star formation process. These winds are thought to efficiently remove the excess angular momentum from disk matter, enabling the flow of material to the centre to form the star. Disk-winds originate from radially extended regions of the disk, and are believed to be accelerated magnetocentrifugally from disk surfaces by the mechanism proposed by Blandford \& Payne (1982). In this scenario, the disk is threaded by large-scale magnetic field lines, which bend radially outwards. If they are sufficiently inclined, matter near the disk surface can be accelerated centrifugally along the field lines (the bead-on-a-wire effect) initiating a large-scale outflow.

To explore the fate of particles embedded in a protostellar wind, we first obtained a radially-localised wind solution for fluid conditions expected to be typical in protostellar disks at a distance of 1 AU from the protostar (see Königl et al. 2010; Salmeron et al. 2011, for the method and parameter space solutions). We then calculated the vertical motion, growth, and thermal processing of spherical, compact dust particles embedded in the fluid (Salmeron \& Ireland 2012a,b). Refractory precursor aggregates are heated while being lifted in the wind, grow through amalgamation, and eventually become heavy enough to drop back to the disk where they may assemble with the matrix. Small particles (radii $\sim 0.01 \mathrm{~cm}$ ) are accelerated away from the disk whereas $\mathrm{cm}$-sized particles sink to the midplane. Intermediate-sized particles (radii $\sim 0.1 \mathrm{~cm}$ ) are initially lifted but, as they grow, gravity causes them to turn back towards the disk. As shown in figure 

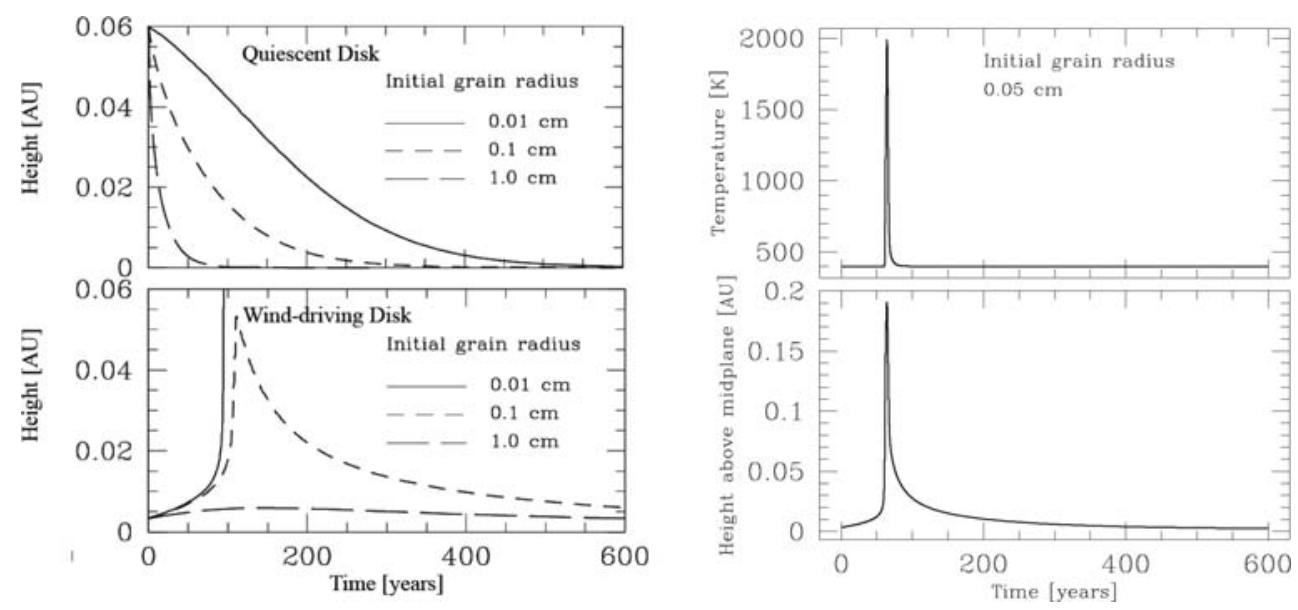

Figure 1. Left panel: Height evolution of dust particles of different sizes embedded in a quiescent or a wind-driving disk. They move through the disk and grow by amalgamation with smaller particles. In a quiescent disk, the particles rapidly settle to the midplane (top, Dullemond \& Dominik 2005; Salmeron \& Ireland 2012b). In a wind-driving disk (bottom panel), very small grains are accelerated away by the outflow, while massive $(\mathrm{cm}$-sized) particles sink back to the midplane. Intermediate-sized grains $(\sim 0.1 \mathrm{~cm})$ are initially lifted but, as they grow, gravity becomes dominant and pulls them back to the disk. Right panel: Evolution of temperature (top panel) and height above the midplane (bottom panel), of a dust particle with an initial radius of $0.05 \mathrm{~cm}$ and a temperature of $400 \mathrm{~K}$, released at $\mathrm{z}=0.003 \mathrm{AU}$ in a typical disk-wind solution (Salmeron \& Ireland 2012a). The peak temperature experienced by the particle is near $2000 \mathrm{~K}$.

1 , we find that such processing at radial distances of $\sim 1-3$ AU can heat precursors to the appropriate temperature to melt chondrules and explain their basic properties, while retaining association with the colder material that provides the matrix.

Processing in a disk wind naturally explains the tight size range of chondrules in a given chondrite, as well as the large proportion of chondrule material present in chondrite meteorites. Formation of compound chondrules is expected as the relative speed between similarly-sized chondrules remains low. Dusty rims can be accreted as chondrules fall back to the disk, and during recycling to the disk interior. Finally, the complementary composition of chondrules and matrix can be maintained, as the processed material remains local to the wind-launching region in the nebula.

\section{References}

Bally, J., Reipurth, B., \& Davis, C. J. 2007, Protostars and Planets V, 215

Blandford, R. D. \& Payne, D. G. 1982, MNRAS, 199, 883

Cabrit, S. 2007, Star-Disk Interaction in Young Stars, 243, 203

Dullemond, C. P. \& Dominik, C. 2005, A \& A, 434, 971

Königl, A., Salmeron, R. \& Wardle, M. 2010, MNRAS, 401(1), 479

Salmeron, R. \& Ireland, T. 2012a, Earth and Planetary Science Letters (2012), 327, 61

Salmeron, R. \& Ireland, T. 2012b, Meteoritics and Planetary Science, 47(12), 1922

Salmeron, R., Königl, A., \& Wardle, M. 2011, MNRAS, 412(2), 1162 